\title{
Uma métrica de roteamento para Internet das Coisas Móveis
}

\author{
Gabriel Augusto R. dos Reis \\ gabriel.requena@aluno.ufop.edu.br \\ Universidade Federal de Ouro Preto \\ (UFOP) \\ João Monlevade, Minas Gerais
}

\author{
Bruno P. Santos \\ bruno.ps@ufop.edu.br \\ Universidade Federal de Ouro Preto \\ (UFOP) \\ João Monlevade, Minas Gerais
}

\author{
Luiz F. M. Vieira \\ lfvieira@dcc.ufmg.br \\ Universidade Federal de Minas Gerais \\ (UFMG) \\ Belo Horizonte, Minas Gerais
}

\begin{abstract}
O processo de roteamento na Internet of Things (IoT) é um aspecto chave quando dispositivos podem mover. O roteamento habilita a comunicação entre os dispositivos, potencializando a funcionalidade dos objetos do dia-a-dia. Contudo, a mobilidade causa frequentes mudanças na topologia tornando o roteamento um desafio. A maioria dos protocolos de roteamento da IoT usam um estimador de qualidade de enlace (Link Quality Estimator (LQE)) no processo de escolha de rotas, sendo o Expected Transmission Count (ETX) um dos mais utilizados para ponderar enlaces sem fio entre dispositivos estáticos. Contudo, o ETX não funciona bem quando os dispositivos se movem. Neste artigo, apresentamos o Mobility aware Expected Transmission Count (MobETX) como estimador para enlaces sem fio entre dispositivos móveis. MobETX mantém as características do ETX, mas usa métricas de mobilidade dos dispositivos para dar assistência na escolha de rotas. Em nossos experimentos, o protocolo de roteamento usando MobETX apresentou melhora de ao menos 5\% na taxa de entrega de pacotes quando comparado com ETX em cenários de alta mobilidade e $>98 \%$ de entrega de pacotes em cenários estáticos.
\end{abstract}

\section{KEYWORDS}

LQE, ETX, MobETX, Estimador de qualidade de enlace, Expected Transmission Count, Mobile Expected Transmission Count

\section{INTRODUÇÃO}

Internet of Things (IoT) é um conceito que vem ganhando força no cenário moderno, sua ideia básica é aprimorar objetos diários através da comunicação (tipicamente sem fio) [1, 9, 11], facilitando tomadas de decisões ou obtenção de respostas a um dado evento.

Para viabilizar o funcionamento da IoT, destacamos a construção e manutenção eficiente das rotas entre os dispositivos, como uma das principais subtarefas de suporte a IoT. O Routing Protocol for Low-Power and Lossy Networks (RPL) [17] é um protocolo de roteamento com suporte ao Internet Protocol version 6 (IPv6) frequentemente utilizado na IoT. O RPL é baseado em vetor de distâncias que utiliza um estimador de qualidade de enlace, referenciados aqui pelo termo em inglês Link Quality Estimator (LQE), que visa classificar os enlaces sem fio em uma determinada janela de tempo.

Em [2] são apresentadas duas categorias de LQEs: Os LQEs baseados em hardware, que obtêm as informações diretamente do transceptor de rádio (por exemplo, o rádio CC2420 [8] e similares) e os

In: I Concurso de Trabalhos de Iniciação Científica (CTIC 2021), Minas Gerais, Brasil Anais Estendidos do Simpósio Brasileiro de Sistemas Multimídia e Web (WebMedia) Porto Alegre: Sociedade Brasileira de Computação, 2021.

(c) 2021 SBC - Sociedade Brasileira de Computação.

ISSN 2596-1683
LQEs baseados em software que aplicam algum método numérico ou estatístico sobre o envio, recepção e/ou confirmação de pacotes. Naturalmente, os LQEs baseados em software são amplamente empregados devido ao seu fácil projeto, implantação e custo associado.

Atualmente, o Expected Transmission Count (ETX) [4] tem notoriedade como LQE devido às suas características que serão citadas na seção 3.1. O ETX tem sido empregado em diferentes implementações de protocolos de roteamento ao longo do tempo [9, 10, 12, 13], além disso, o ETX foi estendido de diferentes modos para acomodar situações onde o ETX original não apresenta bons resultados [3, 5].

Neste trabalho, damos destaque ao cenário móvel. Em redes, especialmente no ponto de vista de roteamento, a mobilidade implica em mudanças frequentes na topologia, que podem requerer atualizações de rotas. Embora já existam diversas métricas de roteamento, atualmente essas métricas apenas qualificam os enlaces sem fio de acordo com o LQE que, no melhor de nossos conhecimentos, não levam em conta as características de mobilidade dos dispositivos.

Diante do acima exposto, a principal contribuição deste trabalho é a proposição de um LQE chamado de Mobility aware Expected Transmission Count (MobETX), o qual incorpora características de mobilidade dos dispositivos ao ETX. O objetivo do MobETX é melhorar a construção de rotas dos protocolos de roteamento (ex.: RPL) mesmo sob eventos de mobilidade dos dispositivos. Este trabalho também introduz o conceito de LQEs híbridos, estendendo a classificação proposta em [2]. Em nossos experimentos, o MobETX apresentou melhora na taxa de entrega de pacotes de ao menos $5 \%$, em situações com dispositivos móveis, quando comparada com outras métricas (ex.: Métricas baseadas em hop-count e ETX). E em cenários estáticos, o MobETX se comporta como o ETX entregando $>98 \%$ dos pacotes quando uma rota existe e sem uso de mecanismos de confirmação.

\section{TRABALHOS RELACIONADOS}

O RPL é um protocolo genérico que se adapta a uma variedade de tipos de rede ao usar diferentes Funções Objetivo (OFs) [15]. Em resumo, uma OF é usada para selecionar nós que serão roteadores (isto é, nós que servem como pais ou possíveis sucessores) em direção à raiz da estrutura criada pelo RPL e para computar o rank de um dispositivo, uma distância lógica entre a raiz e o nó.

Uma OF descreve como um nó selecionará parentes em potencial a partir de sua vizinhança. A escolha do novo pai preferido ( $p p_{\text {novo }}$ ) é feita através da comparação do custo da métrica do possível pai candidato $(p c)$ com o pai preferido atual ( $\left.p p_{\text {atual }}\right)$, tal como descrito na Equação 1 . Observe que é indicado um limiar $(\lambda)$ na Equação 1 que serve como fronteira para a troca de pai preferido.

$$
p p_{\text {novo }}=\left\{\begin{array}{cc}
p c, & s e\left(p c<p p_{\text {atual }}-\lambda\right) . \\
p p_{\text {atual }}, & \text { caso contrário }
\end{array}\right.
$$




\subsection{Classificação dos LQEs}

Diversos LQEs foram propostos ao longo do tempo, em [2], os autores propõem uma classificação para os LQEs até então existentes. A princípio, os LQEs foram classificados como aqueles baseados em hardware ou em software (observe a Figura 1). Nós também concordamos com tal classificação e taxonomia. Porém, vamos além ao estendê-la para acomodar também LQEs recentes, em um grupo que chamamos de LQEs híbridos, isto é, aqueles que estão na interseção entre uso de hardware e software. No melhor de nossos conhecimentos esses LQEs não consideram características de mobilidade.

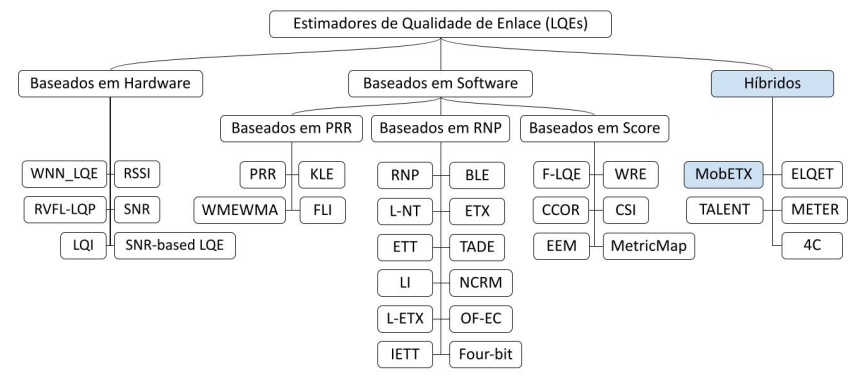

Figura 1: Classificação dos LQEs.

O MobETX se enquadra como LQE híbrido, assim como outras propostas recentes (vide Figura 1). A principal diferença da nossa abordagem, é que o MobETX utiliza métricas de mobilidade para considerar fatores da mobilidade dos dispositivos.

\subsection{Métricas de mobilidade}

Métricas de mobilidade são medidas quantitativas que representam diversos aspectos da mobilidade humana e podem ser extraídas durante a movimentação de um dispositivo. Essas métricas são usadas para analisar o comportamento dos dispositivos ao longo de sua rota. Para aprofundamento sobre métricas de mobilidade recomendamos a leitura de $[14,16]$.

As métricas utilizadas no MobETX são: Velocidade média; Duração média do enlace e Número total de enlaces. Todas as métricas utilizadas se referem do início até o presente momento da rede.

\section{O ESTIMADOR MOBETX}

\subsection{Background}

O ETX, um LQE baseado em software, é um dos LQEs com maior notoriedade atualmente [9]. Ele foi desenvolvido como uma alternativa ao Hop-Count, pois notou-se que em comunicações sem fio, nem sempre a menor rota (em número de saltos) provê o melhor desempenho na comunicação. Neste sentido, o objetivo do ETX é auxiliar no cálculo da rota com o menor número de transmissões e retransmissões, levando em consideração a perda de pacotes e a assimetria dos enlaces sem fio. O ETX é calculado através da equação $E T X=\frac{1}{d_{f} \times d_{r}}$, onde $d_{f}$ é a probabilidade do pacote ser entregue com sucesso e $d_{r}$ é a probabilidade da confirmação ser recebida [4].

Os autores do ETX mostram que os protocolos de roteamento que a usam, possuem maior rendimento em redes sem fio de múltiplos saltos. Isso ocorre, pois o ETX minimiza o número esperado de transmissões e retransmissões, para entregar um pacote com sucesso. As principais características do ETX são: Considerar a taxa de entrega de pacotes bem como assimetria dos enlaces; Penalizar rotas com mais saltos; Minimizar o uso da banda com mensagens de controle desnecessárias e; Diminuir a energia gasta na rede, pois minimiza o número de transmissões por pacote.

\subsection{Um estimador híbrido: MobETX}

Apresentamos aqui o MobETX, um estimador de enlace que se difere dos anteriormente citados, pois utiliza informações da mobilidade do dispositivo, juntamente com o ETX, a fim de estimar a qualidade do enlace, mantendo todas as características do ETX citadas previamente. Projetado para ser utilizado em cenários com padrões de movimentação similar ao humano ou em cenários com grande variação de velocidade nos dispositivos.

Para estimar a mobilidade dos dispositivos, utilizamos métricas de mobilidade, presentes nas Equações 2 e 3, representando a média de tempo de duração dos enlaces realizados pelo dispositivo e a grandeza de sua velocidade média com relação a velocidade máxima (definida a priori) que pode ser alcançada na rede respectivamente, onde (i) é o dispositivo em questão. Na equação $2, n$ é um enlace feito pelo nó $i$, a duração do enlace é obtida através da camada de enlace e $\Omega$ é o conjunto de todos os enlaces feitos pelo nó.

$\mathrm{Na}$ Equação 3, $v_{i}$ avg indica a velocidade média do dispositivo e $v_{\max }$ é a velocidade máxima que os dispositivos podem alcançar na rede. Na Equação 4, temos a Estimativa de Mobilidade (EM), onde $\tau$ se refere ao tempo que o dispositivo está conectado na rede e $\alpha$ $([0,1])$ é responsável por balancear as Equações 2 e 3. É importante observar que estas métricas de mobilidade foram escolhidas devido à sua fácil obtenção pelos nós, baixo custo e processamento e a não necessidade de solicitar dados aos nós vizinhos, o que, poderia acarretar em mais transmissões de controle. Dentre a métricas utilizadas, apenas a velocidade, em $v_{i}$ avg, é obtida através de hardware.

$$
\begin{gathered}
\Delta_{i}=\frac{\sum_{n \in \Omega_{i}} \text { duração } n}{\left|\Omega_{i}\right|} \\
v_{i}=\left\{\begin{array}{cc}
0, & \text { se ve } v_{\text {max }}=0 . \\
\frac{v_{i} \text { avg }}{v_{\max }}, & \text { caso contrário }
\end{array}\right. \\
\text { EMi }_{i}=\left(\left(1-\left(\alpha \cdot\left(\frac{\Delta_{i}}{\tau}\right)\right)\right)+\left((1-\alpha) \cdot v_{i}\right)\right) \\
\operatorname{MobETX}=((\beta \cdot E T X)+((1-\beta) \cdot E M \cdot \gamma))
\end{gathered}
$$

O MobETX é definido na Equação 5 e considera tanto o ETX quanto a EM. Na equação aparece o termo $\beta$, o qual é usado para ajustar o peso entre o ETX e a EM. Essas métricas possuem escalas diferentes, sendo assim $\gamma$ é usado para ajuste da EM.

Para a implementação do MobETX, sugerimos a utilização da Mobile Expected Transmission Count - Objective Function (MobETX$\mathrm{OF}$ ) conforme Equação 6. A MobETX-OF é similar à Minimum Rank Objective Function with Hysteresis (MRHOF) apresentada no RFC6719 [7], mas utilizando-se o MobETX ao invés do ETX. A MobETX-OF seleciona seu nó pai a partir da comparação entre todos os nós de uma lista de possíveis pais, comparando dois a dois, os Ranks destes possíveis pais e escolhendo o menor valor entre eles. Para que haja uma troca eficiente de nó pai, é recomendado que se utilize o limiar mínimo de 8, ou seja, a troca, somente é 
realizada caso o Rank deste seja 8 unidades menor que o Rank do nó pai atual. Após a escolha, o dispositivo calcula seu próprio Rank a partir da Equação 6, onde Rank base é o valor de rank inicial de cada nó e Rank $k_{\text {incremento }}$ é o valor adicionado a cada salto para evitar laços de roteamento. É recomendado que os valores de Rank $k_{\text {base }}$

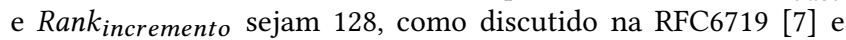
definido como padrão do Contiki-OS. Após a definição do Rank atual do nó, este valor é divulgado a todos os nós vizinhos.

$$
\text { Rank }=\text { Rank }_{\text {base }}+\text { Rank }_{\text {incremento }}+\text { MobETX }
$$

\section{MATERIAIS E MÉTODOS}

Nesta seção iremos abordar os métodos utilizados para realizar os experimentos e a avaliação dos resultados obtidos. Como ambiente de experimentação foi usado o emulador de redes Cooja presente no Contiki-OS 3.0 [6]. Os parâmetros utilizados para simulação são descritos na Tabela 1, demais parâmetros foram mantidos como no padrão do sistema Contiki-OS. Para estudar o efeito da mobilidade na taxa de entrega de dados usando os estimadores MobETX e ETX, usamos um esquema de experimento fatorial $2^{k}$.

\begin{tabular}{lr}
\hline \multicolumn{2}{c}{ Parâmetros do Contiki/Cooja } \\
\hline \# de nós & 100 \\
Modelo de conectividade & UDGM \\
Dimensão & $1000 \mathrm{~m}^{2}$ \\
Tipos de nós & Cooja Mote \\
Protocolos & RPL, Trickle \\
Intervalo de transmissão de pacotes & $300 \mathrm{~s}$ \\
Randomização de transmissão de pacotes & $300 \mathrm{~s}$ \\
Tempo de simulação & $24 \mathrm{~h}$ \\
\hline
\end{tabular}

Tabela 1: Tabela com os parâmetros do Contiki/Cooja.

A mobilidade dos dispositivos é de fato um fator que influencia no desempenho das métricas que estimam a qualidade dos enlaces, sendo, portanto, um fator que será considerado. Ademais, na confecção do MobETX, três outros fatores possuem destaque e podem impactar no seu desempenho, sendo eles: $\mathrm{O}$ limiar $\lambda$ para troca do pai preferido (vide Equação 1), o $\alpha$ da Equação 4 que ajusta o compromisso entre média de duração dos enlaces e velocidade do dispositivo, e, por fim, o $\beta$ da Equação 5, que faz o balanço do compromisso entre usar ETX e EM. Com base nesses 4 fatores $(k=4)$, foram realizados 16 experimentos, sendo 3 repetições "aleatórias" para os de cenário estático e 2 repetições para os de cenário móvel, diferença essa, devido ao tempo de execução necessários para cada experimento. As configurações de cada experimento variaram da seguinte forma: $\lambda$ assumindo os valores de 8 ou 16, $\alpha$ assumindo os valores de 0,3 ou 0,7 e $\beta$ assumindo 0,5 ou 0,9 . Como modelos de mobilidade dos cenários de experimentação foram utilizados o Random Waypoint, que representa mobilidade extrema e cenários estáticos, ou seja, de mobilidade nula. Esses cenários foram escolhidos propositalmente para verificar os limites da abordagem proposta. Desta maneira, espera-se que em cenários de mobilidade moderada o MobETX venha a apresentar resultados satisfatórios.

\subsection{Resultados}

Esta seção apresenta os resultados obtidos a partir dos experimentos fatoriais $2^{k}$. Ademais, nas Tabelas 2 e 3 são apresentadas as interações entre os fatores estudados e com isto, como cada um interfere no resultado final do experimento. As colunas $\lambda$, $\alpha$ e $\beta$, representam as variáveis de configuração das simulações ${ }^{1}$ e a coluna $A V G$ representa a média dos resultados obtidos nos experimentos configurados de acordo com $\lambda, \alpha$ e $\beta$. A última linha das tabelas apresenta o Valor de interação, o qual representa o quanto uma determinada variável ou conjunto delas impacta nos resultados obtidos. Damos destaque para o $\alpha$ e $\beta$ que apresentam os valores mais significativos (realçado nas tabelas), sendo, portanto os fatores que mais afetam os resultados, o que é justo pois, são exatamente os fatores que ajustam os compromissos referentes à mobilidade das entidades da rede e o balanceamento entre o ETX e a EM.

\begin{tabular}{|c|c|c|c|c|c|c|c|c|c|c|}
\hline$\lambda$ & $\alpha$ & $\beta$ & AVG & $\lambda$ & $\alpha$ & $\beta$ & $\lambda \alpha$ & $\lambda \beta$ & $\alpha \beta$ & $\lambda \alpha \beta$ \\
\hline-1 & -1 & -1 & 87,94 & $-87,9$ & $-87,9$ & $-87,9$ & 87,94 & 87,94 & 87,94 & $-87,9$ \\
\hline 1 & -1 & -1 & 88,75 & 88,75 & $-88,8$ & $-88,8$ & $-88,8$ & $-88,8$ & 88,75 & 88,75 \\
\hline-1 & -1 & 1 & 82,44 & $-82,4$ & $-82,4$ & 82,44 & 82,44 & $-82,4$ & $-82,4$ & 82,44 \\
\hline 1 & -1 & 1 & 82,88 & 82,88 & $-82,88$ & 82,88 & $-82,88$ & 82,88 & $-82,88$ & $-82,88$ \\
\hline-1 & 1 & -1 & 90,15 & $-90,2$ & 90,15 & $-90,2$ & $-90,2$ & 90,15 & $-90,2$ & 90,15 \\
\hline 1 & 1 & -1 & 91,53 & 91,53 & 91,53 & $-91,5$ & 91,53 & $-91,5$ & $-91,5$ & $-91,5$ \\
\hline-1 & 1 & 1 & 83,31 & $-83,3$ & 83,31 & 83,31 & $-83,3$ & $-83,3$ & 83,31 & $-83,3$ \\
\hline 1 & 1 & 1 & 83,42 & 83,42 & 83,42 & 83,42 & 83,42 & 83,42 & 83,42 & 83,42 \\
\hline \multicolumn{4}{|c|}{ Valor de interação } & 0,68 & 1,59 & $-6,57$ & 0,06 & $-0,4$ & $-0,89$ & $-0,22$ \\
\hline
\end{tabular}

Tabela 2: Matriz de efeito dos fatores em cenários móveis.

\begin{tabular}{|c|c|c|c|c|c|c|c|c|c|c|}
\hline$\lambda$ & $\alpha$ & $\beta$ & AVG & $\lambda$ & $\alpha$ & $\beta$ & $\lambda \alpha$ & $\lambda \beta$ & $\alpha \beta$ & $\lambda \alpha \beta$ \\
\hline-1 & -1 & -1 & 1,43 & $-1,43$ & $-1,43$ & $-1,43$ & 1,43 & 1,43 & 1,43 & $-1,43$ \\
\hline 1 & -1 & -1 & 1,48 & 1,48 & $-1,48$ & $-1,48$ & $-1,48$ & $-1,48$ & 1,48 & 1,48 \\
\hline-1 & -1 & 1 & 1,44 & $-1,44$ & $-1,44$ & 1,44 & 1,44 & $-1,44$ & $-1,44$ & 1,44 \\
\hline 1 & -1 & 1 & 1,30 & 1,30 & $-1,30$ & 1,30 & $-1,30$ & 1,30 & $-1,30$ & $-1,30$ \\
\hline-1 & 1 & -1 & 3,41 & $-3,41$ & 3,41 & $-3,41$ & $-3,41$ & 3,41 & $-3,41$ & 3,41 \\
\hline 1 & 1 & -1 & 4,72 & 4,72 & 4,72 & $-4,72$ & 4,72 & $-4,72$ & $-4,72$ & $-4,72$ \\
\hline-1 & 1 & 1 & 1,39 & $-1,39$ & 1,39 & 1,39 & $-1,39$ & $-1,39$ & 1,39 & $-1,39$ \\
\hline 1 & 1 & 1 & 1,30 & 1,30 & 1,30 & 1,30 & 1,30 & 1,30 & 1,30 & 1,30 \\
\hline \multicolumn{4}{|c|}{ Valor de interacão } & 0,28 & 1,29 & $-1,40$ & 0,32 & $-0,39$ & $-1,31$ & $-0,30$ \\
\hline
\end{tabular}

Tabela 3: Matriz de efeito dos fatores em cenários estáticos.

Para verificar se a variação de $\lambda, \alpha$ e $\beta$ são significativos nos resultados obtidos, construímos as Tabelas 4 e 5 ANOVA. A tabela ANOVA tem como objetivo fazer uma análise estatística dos efeitos dos fatores em análise, a partir dos resultados dos experimentos.

Nas Tabelas 4 e 5, a razão F é o resultado obtido na ANOVA a fim de ser comparado com o valor na tabela $\mathrm{F}$ e nos informar se este valor é estatisticamente significativo. Em nossos experimentos, ao se utilizar uma significância de $5 \%$ para ambos os cenários, obtemos na tabela $\mathrm{F}$, o valor de 5,32 no cenário estático e 4,46 para o cenário móvel. Com estes valores, concluímos que a variação de $\alpha, \beta$ e de sua interação são relevantes no cenário estático e em cenário móvel, a variação de $\alpha$ e $\beta$ tem efeito significativo em nossos resultados (em destaque nas tabelas). Concluímos, por fim, que $\alpha$ e $\beta$ tem grande relevância em ambos os casos, e, portanto, em trabalhos futuros, devem ser considerados na elaboração de novos LQEs similares.

Após observação das tabelas ANOVA e dos resultados obtidos, a configuração padrão foi escolhida, com os valores de $\lambda=16$, $\alpha=0.3$ e $\beta=0.9$. A partir desta configuração foram realizados 15 experimentos com ambos LQEs a fim de verificar a variância dos resultados e analisar a viabilidade do MobETX. Os resultados destes experimentos podem ser visualizados nas Figuras 2(a) e 2(b).

Para finalizar os gráficos de comparação, as Figuras 2(c) e 2(d) apresentam os intervalos de confiança obtidos nos experimentos, com uma confiança de 95\% (distribuição T-Student). Nestes gráficos

${ }^{1}$ Variável em +1 para o maior valor e -1 para o menor valor possível para a determinada variável conforme variação citada anteriormente 


\begin{tabular}{lcccc}
\hline Fator & Soma dos quadrados & graus de liberdade & Quadrado médio & Razão F \\
\hline$\lambda$ & 2,7973677 & 2 & 1,3986838 & 1,0701165 \\
$\alpha$ & 259,6240735 & 2 & 129,8120367 & 99,3176565 \\
$\beta$ & 15,3482521 & 2 & 7,6741260 & 5,8713832 \\
$\lambda \alpha$ & 0,9998594 & 2 & 0,4999297 & 0,3824903 \\
$\lambda \beta$ & 0,0239234 & 2 & 0,0119617 & 0,0091517 \\
$\alpha \beta$ & 4,7842652 & 2 & 2,3921326 & 1,8301924 \\
$\lambda \alpha \beta$ & 0,2991444 & 2 & 0,1495722 & 0,1144359 \\
Erro & 10,4563108 & 8 & 1,3070388 & \\
Total & 294,3331969 & 22 & & \\
\hline
\end{tabular}

Tabela 4: Tabela ANOVA em cenário móvel.

\begin{tabular}{lrrrr}
\hline Fator & Soma dos quadrados & graus de liberdade & Quadrado médio & Razão F \\
\hline$\lambda$ & 0,3195881 & 1 & 0,3195881 & 0,2906174 \\
$\alpha$ & 7,8822810 & 1 & 7,8822810 & $7, \mathbf{1 6 7 7 5 0 8}$ \\
$\beta$ & 6,6748314 & 1 & 6,6748314 & $\mathbf{6 , 0 6 9 7 5 6 7}$ \\
$\lambda \alpha$ & 0,6306541 & 1 & 0,6306541 & 0,5734852 \\
$\lambda \beta$ & 0,4278915 & 1 & 0,4278915 & 0,3891030 \\
$\alpha \beta$ & 6,9072746 & 1 & 6,9072746 & $\mathbf{6 , 2 8 1 1 2 8 9}$ \\
$\lambda \alpha \beta$ & 0,3636942 & 1 & 0,3636942 & 0,3307253 \\
Erro & 8,7974944 & 8 & 1,0996868 & \\
Total & 32,0037093 & 15 & & \\
\hline
\end{tabular}

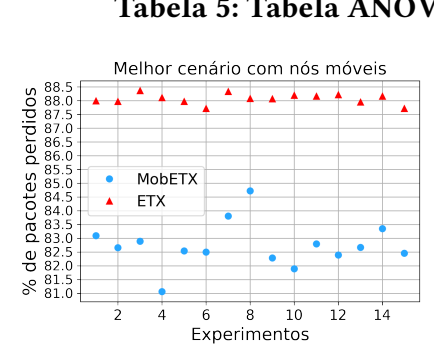

(a)

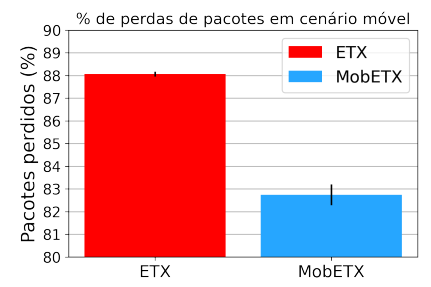

(c)

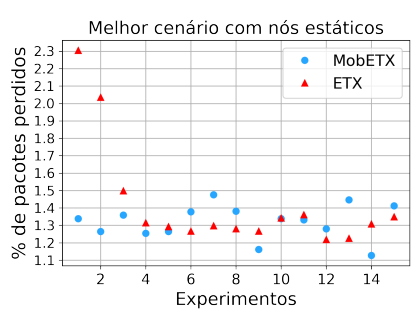

(b)

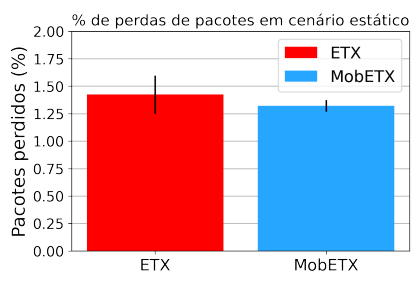

(d)
Figura 2: Experimentos na melhor configuração.

podemos notar que o MobETX em um cenário móvel apesar de, uma variância maior entre seus resultados (variando 0,91 enquanto o ETX varia 0,20 pontos percentuais), possui uma melhora de aproximadamente $5 \%$ em relação à porcentagem de perda de pacotes, quando comparado aos resultados obtidos pelo ETX. Em cenário estático, mantém performance similar ao ETX (média de 1,32\% para o MobETX e $1,42 \%$ do ETX).

\section{CONCLUSÃO}

Um novo estimador, chamado MobETX, foi apresentado neste artigo. O MobETX, categorizado como um LQE híbrido, estima a qualidade do enlace utilizando métricas de mobilidade dos nós da rede em conjunto com o ETX para se obter uma melhora na taxa de entrega de pacotes da rede. Foram feitos experimentos, baseados no experimento fatorial $2^{k}$ para definir os valores adequados dos fatores de configuração. Após, foram realizados experimentos a partir da configuração escolhida em cenários com dispositivos móveis e em cenários com dispositivos estáticos, comparando os resultados obtidos com o desempenho do ETX nos mesmos cenários.

Os resultados obtidos indicam que o MobETX superou o ETX em $5 \%$ na taxa de perda de pacotes em cenários com dispositivos móveis, com performance similar em redes com dispositivos estáticos. Estes resultados mostram que o MobETX pode ser uma alternativa promissora ao ETX, visto que além da melhora na perda de pacotes, mantém todas as características do ETX, sem a necessidade de alterações no protocolo de roteamento subjacente como, por exemplo, o RPL.

Futuramente, o MobETX necessitará de experimentos mais complexos de mobilidade como, por exemplo, cenários com múltiplos padrões de mobilidade simultâneos. Outro aprimoramento futuro é adaptar o MobETX para fazer a estimativa de velocidade baseado na força do sinal recebido, caso a opção de hardware esteja indisponível. Ademais, destaca-se validar o MobETX em cenários reais de testbed dado que sua implementação é compatível com o ContikiOS. O MobETX pode ser incorporado com outros LQEs já existentes, a fim de otimizar outros fatores, aqui não abordados, como consumo energético da rede, latência, centralidade, entre outros.

\section{REFERENCES}

[1] Luigi Atzori, Antonio Iera, and Giacomo Morabito. 2010. The internet of things: A survey. Computer networks 54, 15 (2010), 2787-2805.

[2] Nouha Baccour, Anis Koubâa, Luca Mottola, Marco Antonio Zúñiga, Habib Youssef, Carlo Alberto Boano, and Mário Alves. 2012. Radio link quality estimation in wireless sensor networks: A survey. ACM TOSN 8, 4 (2012), 34.

[3] Michael Bahr, Jianping Wang, and Xiaohua Jia. 2006. Routing in Wireless Mesh Networks. Proceedings of the Annual International Conference on MOBICOM (2006). https://doi.org/10.1201/9781420013542.ch4

[4] Douglas SJ De Couto, Daniel Aguayo, John Bicket, and Robert Morris. 2005. A high-throughput path metric for multi-hop wireless routing. Wireless networks (2005).

[5] Richard Draves, Jitendra Padhye, and Brian Zill. 2004. Comparison of routing metrics for static multi-hop wireless networks. ACM SIGCOMM CCR (2004)

[6] Adam Dunkels, Bjorn Gronvall, and Thiemo Voigt. 2004. Contiki-a lightweight and flexible operating system for tiny networked sensors. In 29th annual IEEE $L C N$.

[7] Omprakash Gnawali and Philip Levis. 2012. The minimum rank with hysteresis objective function. RFC 6719 (2012).

[8] Texas Instruments. 2007. CC2420 datasheet. Reference SWRS041B (2007)

[9] Wafa'a Kassab and Khalid A Darabkh. 2020. A-Z survey of Internet of Things: Architectures, protocols, applications, recent advances, future directions and recommendations. Journal of Network and Computer Applications 163 (2020), 102663.

[10] Bruno P Santos, Olga Goussevskaia, Luiz FM Vieira, Marcos AM Vieira, and Antonio AF Loureiro. 2018. Mobile matrix: routing under mobility in IoT, IoMT, and social IoT. Ad Hoc Networks 78 (2018), 84-98.

[11] Bruno P Santos, Lucas AM Silva, CSFS Celes, João B Borges, Bruna S Peres Neto, Marcos Augusto M Vieira, Luiz Filipe M Vieira, Olga N Goussevskaia, and A Loureiro. 2016. Internet das coisas: da teoria à prática. Minicursos SBRC (2016).

[12] Bruno P Santos, Luiz FM Vieira, and Marcos AM Vieira. 2017. CGR: Centralitybased green routing for Low-power and Lossy Networks. Computer Networks (2017).

[13] Bruno Pereira Santos, Luiz Filipe Menezes Vieira, and Antonio Alfredo Ferreira Loureiro. 2020. Routing and Mobility Management in the Internet of Things. In Anais Estendidos do XXXVIII SBRC. SBC, 161-168.

[14] Fabrício R Souza, Augusto CSA Domingues, Pedro O Vaz de Melo, and Antonio AF Loureiro. 2018. MOCHA: Um framework para caracterização e comparação de traces de mobilidade. In Anais do XXXVI SBRC. SBC.

[15] Pascal Thubert et al. 2012. Objective function zero for the routing protocol for low-power and lossy networks (RPL). (2012).

[16] University of Osnabrück 2016. A Mobility Scenario Generation and Analysis Tool. University of Osnabrück. http://sys.cs.uos.de/bonnmotion/doc/README.pdf.

[17] Tim Winter, Pascal Thubert, Anders Brandt, Jonathan W Hui, Richard Kelsey, Philip Levis, Kris Pister, Rene Struik, Jean-Philippe Vasseur, Roger K Alexander, et al. 2012. RPL: IPv6 Routing Protocol for Low-Power and Lossy Networks. $r f c$ 6550 (2012), 1-157. 